\title{
Two binary Darboux transformations for the KdV hierarchy with self-consistent sources
}

\author{
Yunbo Zeng $\dagger \quad$ Wen-Xiu Ma $\ddagger$ Yijun Shao $\dagger$ \\ $\dagger$ Department of Mathematical Sciences, Tsinghua University, Beijing 100084, China \\ $\ddagger$ Department of Mathematics, City University of Hong Kong, Kowloon, Hong Kong, China
}

\begin{abstract}
Two binary (integral type) Darboux transformations for the KdV hierarchy with self-consistent sources are proposed. In contrast with the Darboux transformation for the KdV hierarchy, one of the two binary Darboux transformations provides non auto-Bäcklund transformation between two n-th KdV equations with self-consistent sources with different degrees. The formula for the m-times repeated binary Darboux transformations are presented. This enables us to construct the N-soliton solution for the KdV hierarchy with self-consistent sources.
\end{abstract}

Keywords: binary Darboux transformation, KdV hierarchy with self-consistent sources, Lax representation, N-soliton solution

\section{Introduction}

The soliton equations with self-consistent sources have important physical applications (see [1]-11]), for example, the KdV equation with self-consistent source describes the interaction of long and short capillary-gravity waves [5. There are some ways to derive the integrable nonlinear evolution equations with self-consistent sources [1, 2, [2, 12, 13]. In recent years soliton equations with self-consistent sources (SESCS) were studied based on the constrained flows of soliton equations which are just the stationary equations of SESCSs [14][19]. Since the Lax representations for the constrained flows of soliton equations can always be deduced from the adjoint representations of the Lax representations for soliton equations, this approach provides a simple and natural way to derive both the SESCSs and their Lax 
representations [15, 16, 17]. The SESCS is an infinite-dimensional integrable Hamiltonian system possessing $t$-type Hamiltonian or bi-Hamiltonian formulation [20] and can be solved by the inverse scattering method [1, 2, 3, 21, 22, 23].

The Darboux transformation is a power tool for solving soliton equations (see [24] for a review). The Darboux transformation for KdV hierarchy was widely studied (see, for example, [24]-27]). In the present paper we will generalize these results to the KdV hierarchy with self-consistent sources. We construct one Darboux transformation and two binary (integral type) Darboux transformations for the KdV hierarchy with self-consistent sources. The Darboux transformations usually present auto-Bäcklund transformations for soliton equations. In contrast with the case of soliton equations, one binary Darboux transformation in our case is proved to be a non auto-Bäcklund transformation between two n-th KdV equations with self-consistent sources with different degrees. This provides an interesting example for constructing non auto-Bäcklund transformations by means of Darboux transformations. Furthermore we present the formula for the m-times repeated binary Darboux transformations and construct the N-soliton solution for the KdV hierarchy with self-consistent sources.

The paper is organized as follows. In the next section we recall the $\mathrm{KdV}$ hierarchy with self-consistent sources and briefly describe how to derive their Lax representation from the adjoint representation of the Lax representation for the KdV hierarchy. In section 3, we briefly review Darboux transformations for the KdV hierarchy and present two binary Darboux transformations. Based on these results, in section 4, we propose one Darboux transformation and two binary Darboux transformations for the KdV hierarchy with selfconsistent sources and show that the first binary Darboux transformation gives the autoBäcklund transformation for the KdV hierarchy with self-consistent sources, and the second binary Darboux transformation leads to a non auto-Bäcklund transformation relating two n-th KdV equations with self-consistent sources with different degrees. Finally in the last section we present the m-times repeated binary Darboux transformations and construct the $\mathrm{N}$-soliton solution for the n-th KdV equation with self-consistent sources.

\section{The KdV hierarchy with self-consistent sources}

To make the paper self-contained, we first recall the high-order constrained flows of the KdV hierarchy and briefly describe how to derive the Lax representation for the $\mathrm{KdV}$ hierarchy with self-consistent sources.

Consider the Schrödinger equation

$$
\phi_{x x}+(\lambda+u) \phi=0
$$


In order to derive the Lax representation for the KdV hierarchy with self-consistent sources, we rewrite the equation (2.1) in the matrix form

$$
\left(\begin{array}{c}
\phi \\
\phi_{x}
\end{array}\right)_{x}=U\left(\begin{array}{c}
\phi \\
\phi_{x}
\end{array}\right), \quad U=\left(\begin{array}{cc}
0 & 1 \\
-\lambda-u & 0
\end{array}\right) .
$$

The adjoint representation of (2.2) reads 28]

$$
V_{x}=[U, V] \equiv U V-V U
$$

Set

$$
V=\sum_{i=0}^{\infty}\left(\begin{array}{cc}
a_{i} & b_{i} \\
c_{i} & -a_{i}
\end{array}\right) \lambda^{-i}
$$

Eq. (2.3) yields

$$
\begin{gathered}
a_{0}=b_{0}=0, \quad c_{0}=-1, \quad a_{1}=0, \quad b_{1}=1, \quad c_{1}=-\frac{1}{2} u \\
a_{2}=\frac{1}{4} u_{x}, \quad b_{2}=-\frac{1}{2} u, \quad c_{2}=\frac{1}{8}\left(u_{x x}+u^{2}\right), \ldots
\end{gathered}
$$

and in general for $k=1,2, \ldots$,

$$
a_{k}=-\frac{1}{2} b_{k, x}, \quad b_{k+1}=L b_{k}=-\frac{1}{2} L^{k-1} u, \quad c_{k}=-\frac{1}{2} b_{k, x x}-b_{k+1}-b_{k} u,
$$

where

$$
L=-\frac{1}{4} \partial^{2}-u+\frac{1}{2} \partial^{-1} u_{x}, \quad \partial=\frac{\partial}{\partial x}, \quad \partial \partial^{-1}=\partial^{-1} \partial=1 .
$$

Set

$$
V^{(n)}=\sum_{i=0}^{n+1}\left(\begin{array}{cc}
a_{i} & b_{i} \\
c_{i} & -a_{i}
\end{array}\right) \lambda^{n+1-i}+\left(\begin{array}{cc}
0 & 0 \\
b_{n+2} & 0
\end{array}\right)
$$

and take

$$
\left(\begin{array}{c}
\phi \\
\phi_{x}
\end{array}\right)_{t_{n}}=V^{(n)}(u, \lambda)\left(\begin{array}{c}
\phi \\
\phi_{x}
\end{array}\right)
$$

or equivalently

$$
\phi_{t_{n}}=A^{(n)}(u, \lambda) \phi, \quad A^{(n)}(u, \lambda) \equiv \sum_{i=0}^{n+1}\left(a_{i}+b_{i} \partial\right) \lambda^{n+1-i}
$$


Then the compatibility condition of Eqs (2.1) and (2.8) or (2.2) and (2.7) gives rise to the KdV hierarchy

$$
u_{t_{n}}=K_{n}[u] \equiv \partial \frac{\delta H_{n}}{\delta u} \equiv-2 b_{n+2, x}, \quad n=0,1, \cdots
$$

where $H_{n}=\frac{4 b_{n+3}}{2 n+3}$. We have

$$
\frac{\delta \lambda}{\delta u}=\phi^{2}, \quad L \phi^{2}=\lambda \phi^{2}
$$

The high-order constrained flows of the KdV hierarchy consist of the equations obtained from the spectral problem (2.1) for $N$ distinct $\lambda_{j}$ and the restriction of the variational derivatives for the conserved quantities $H_{n}$ and $\lambda_{j}$ 229

$$
\begin{gathered}
D\left[\frac{\delta H_{n}}{\delta u}-2 \alpha \sum_{j=1}^{N} \frac{\delta \lambda_{j}}{\delta u}\right] \equiv D\left[-2 b_{n+2}-2 \alpha \sum_{j=1}^{N} \phi_{j}^{2}\right]=0 \\
\phi_{j, x x}+\left(\lambda_{j}+u\right) \phi_{j}=0, \quad j=1, \cdots, N
\end{gathered}
$$

where $n=0,1, \cdots$. According to Eqs (2.5), (2.10) and (2.11), we may define

$$
\begin{gathered}
\widetilde{a}_{i}=a_{i}, \quad \widetilde{b}_{i}=b_{i}, \quad \widetilde{c}_{i}=c_{i}, \quad i=0,1, \ldots, n+1, \\
\widetilde{b}_{n+2+i}=-\alpha \sum_{j=1}^{N} \lambda_{j}^{i} \phi_{j}^{2}, \quad \widetilde{a}_{n+2+i}=-\frac{1}{2} \widetilde{b}_{n+2+i, x}=\alpha \sum_{j=1}^{N} \lambda_{j}^{i} \phi_{j} \phi_{j, x}, \quad i=0,1,2, \ldots, \\
\widetilde{c}_{n+2+i}=-\frac{1}{2} \widetilde{b}_{n+2+i, x x}-\widetilde{b}_{n+3+i}-\widetilde{b}_{n+2+i} u=\alpha \sum_{j=1}^{N} \lambda_{j}^{i} \phi_{j, x}^{2} .
\end{gathered}
$$

Then the construction of $\widetilde{a}_{i}, \widetilde{b}_{i}, \widetilde{c}_{i}$ ensures that

$$
\begin{gathered}
N^{(n)}=\lambda^{n+1} \sum_{k=0}^{\infty}\left(\begin{array}{cc}
\widetilde{a}_{k} & \widetilde{b}_{k} \\
\widetilde{c}_{k} & -\widetilde{a}_{k}
\end{array}\right) \lambda^{-k}+\left(\begin{array}{cc}
\eta & 0 \\
0 & \eta
\end{array}\right) \\
=\sum_{k=0}^{n+1}\left(\begin{array}{cc}
a_{k} & b_{k} \\
c_{k} & -a_{k}
\end{array}\right) \lambda^{n+1-k}+\left(\begin{array}{cc}
\eta & 0 \\
0 & \eta
\end{array}\right)+\alpha \sum_{j=1}^{N} \frac{1}{\lambda-\lambda_{j}}\left(\begin{array}{cc}
\phi_{j} \phi_{j, x} & -\phi_{j}{ }^{2} \\
\phi_{j, x}^{2} & -\phi_{j} \phi_{j, x}
\end{array}\right),
\end{gathered}
$$

where $\eta$ is a constant, also satisfies the adjoint representation (2.3), i.e.

$$
N_{x}^{(n)}=\left[U, N^{(n)}\right]
$$

which gives rise to the Lax representation of the constrained flow (2.11). 
The KdV hierarchy with self-consistent sources is given by [16, 17]

$$
\begin{gathered}
u_{t_{n}}=D\left[\frac{\delta H_{n}}{\delta u}-2 \alpha \sum_{j=1}^{N} \frac{\delta \lambda_{j}}{\delta u}\right] \equiv D\left[-2 b_{n+2}-2 \alpha \sum_{j=1}^{N} \phi_{j}^{2}\right], \\
\phi_{j, x x}+\left(\lambda_{j}+u\right) \phi_{j}=0, \quad j=1, \cdots, N .
\end{gathered}
$$

Since the high-order constrained flows (2.11) are just the stationary equations of the $\mathrm{KdV}$ hierarchy with self-consistent sources $(2.13)$, it is obvious that the zero-curvature representation for the KdV hierarchy with self-consistent sources (2.13) is given by

$$
U_{t_{n}}-N_{x}^{(n)}+\left[U, N^{(n)}\right]=0
$$

with the auxiliary linear problems

$$
\left(\begin{array}{c}
\psi \\
\psi_{x}
\end{array}\right)_{x}=U\left(\begin{array}{c}
\psi \\
\psi_{x}
\end{array}\right), \quad\left(\begin{array}{c}
\psi \\
\psi_{x}
\end{array}\right)_{t_{n}}=N^{(n)}\left(\begin{array}{c}
\psi \\
\psi_{x}
\end{array}\right),
$$

or equivalently

$$
\begin{gathered}
\psi_{x x}+(\lambda+u) \psi=0 \\
\psi_{t_{n}}=A^{(n)} \psi+\eta \psi+\alpha \sum_{j=1}^{N} \frac{1}{\lambda-\lambda_{j}} \phi_{j}\left(\phi_{j, x} \psi-\phi_{j} \psi_{x}\right) .
\end{gathered}
$$

Let assume that all products $\phi_{j} \psi$ decay at $x=-\infty$ and that $\partial^{-1}=\int_{-\infty}^{x} \cdot d x$. It is easy to find from (2.13b) and (2.16a) that

$$
\frac{1}{\lambda-\lambda_{j}}\left(\phi_{j, x} \psi-\phi_{j} \psi_{x}\right)=\partial^{-1} \phi_{j} \psi
$$

Let denote

$$
B_{N}=\alpha \sum_{j=1}^{N} \phi_{j} \partial^{-1} \phi_{j}
$$

Then equation (2.16b) can be rewritten as

$$
\psi_{t_{n}}=Q^{(n, N)} \psi \equiv A^{(n)} \psi+\eta \psi+B_{N} \psi
$$

When $n=1$, the equation (2.13) gives the $\mathrm{KdV}$ equation with self-consistent sources

$$
u_{t_{1}}=-\frac{1}{4}\left(6 u u_{x}+u_{x x x}\right)-2 \alpha D \sum_{j=1}^{N} \phi_{j}{ }^{2}
$$




$$
\phi_{j, x x}+\left(\lambda_{j}+u\right) \phi_{j}=0, \quad j=1, \cdots, N
$$

and the auxiliary linear problem reads

$$
\begin{gathered}
\psi_{x x}+(\lambda+u) \psi=0 \\
\psi_{t_{1}}=\left(\frac{1}{4} u_{x}+\eta\right) \psi+\left(\lambda-\frac{1}{2} u\right) \psi_{x}+\alpha \sum_{j=1}^{N} B_{j} \psi .
\end{gathered}
$$

\section{The Darboux transformation for the KdV hierarchy}

In this section we recall the Darboux transformation for the KdV hierarchy (see [24] for a review, 25, 26, 27]).

(1) The Darboux transformation for the KdV hierarchy.

Assume that $u$ be the solution of the n-th $\mathrm{KdV}$ equation (2.9) and denote the fixed solution of (2.1) and (2.8) with $\lambda=\xi$ by $f=f(x, t, \xi)$. The Darboux transformation (DT) is defined by

$$
\begin{gathered}
\tilde{\phi}=\phi_{x}-\frac{f_{x}}{f} \phi, \\
\tilde{u}=u+2 \partial^{2} \ln f .
\end{gathered}
$$

It is known that the Schrödinger equation (2.1) and (2.8) are covariant with respect to the action of the Darboux transformation (3.1), namely $\tilde{\phi}, \tilde{u}$ satisfy

$$
\begin{gathered}
\tilde{\phi}_{x x}+(\lambda+\tilde{u}) \tilde{\phi}=0, \\
\tilde{\phi}_{t_{n}}=\tilde{A}^{(n)} \tilde{\phi} \equiv A^{(n)}(\tilde{u}, \lambda) \tilde{\phi}
\end{gathered}
$$

and $\tilde{u}$ satisfies the n-th KdV equation (2.9). Eqs. (2.8), (3.1) and (3.3) imply that

$$
\tilde{\phi}_{t_{n}}=\left[\phi_{x}-\frac{f_{x}}{f} \phi\right]_{t_{n}}=\left(A^{(n)} \phi\right)_{x}-\left(\frac{A^{(n)} f}{f}\right)_{x} \phi-\frac{f_{x}}{f} A^{(n)} \phi=\tilde{A}^{(n)} \tilde{\phi} .
$$

So the covariance of (2.1) and (2.8) with respect to the action of DT (3.1) leads to the following lemma.

Lemma 3.1 If $u$ is the solution of the $n$-th $K d V$ equation (2.9 and $f$ is a solution of 2.1) and (2.8) with $\lambda=\xi$ and the Darboux transformation is given by (3.1), then the formula (3.4) holds. 
We now construct the binary Darboux transformation.

(2) The first binary Darboux transformation.

Also, it is known that the linearly independent solution of (2.1) and (2.8) with $\lambda=\xi$ is given by the Liouville formula

$$
g=f \partial^{-1} \frac{1}{f^{2}} .
$$

The DT (3.1) implies that

$$
\tilde{g}=g_{x}-\frac{f_{x}}{f} g=\frac{1}{f}
$$

is one of solutions of (3.2) and (3.3) with $\lambda=\xi$ and $\tilde{u}$ given by (3.1b). The linearly independent solution $\tilde{g}_{1}$ of (3.2) and (3.3) with $\lambda=\xi$ is once more given by the Liouville formula

$$
\tilde{g}_{1}=\tilde{g} \partial^{-1} \frac{1}{\tilde{g}^{2}}=\frac{1}{f} \partial^{-1} f^{2} .
$$

By using $f$ and $\tilde{g}_{1}$, performing two-times repeated DT of (3.1) (notice that the right side of (3.1a) can be added a constant factor) and using (2.17) give rise to the binary Darboux transformation

$$
\begin{gathered}
\bar{\phi}=\frac{1}{\xi-\lambda}\left[\tilde{\phi}_{x}-\frac{\tilde{g}_{1 x}}{\tilde{g}_{1}} \tilde{\phi}\right] \\
=\frac{1}{\xi-\lambda}\left[\xi \phi-\lambda \phi+\frac{f}{\partial^{-1} f^{2}}\left(f_{x} \phi-f \phi_{x}\right)\right]=\phi-\frac{f}{\partial^{-1} f^{2}} \partial^{-1}(f \phi), \\
\bar{u}=\tilde{u}+2 \partial^{2} \ln \tilde{g}_{1}=u+2 \partial^{2} \ln \left(\partial^{-1} f^{2}\right) .
\end{gathered}
$$

Obviously, the equation (2.1) and (2.8) are covariant with respect to the action of the binary DT (3.8), namely $\bar{\phi}, \bar{u}$ satisfy

$$
\begin{gathered}
\bar{\phi}_{x x}+(\lambda+\bar{u}) \bar{\phi}=0, \\
\bar{\phi}_{t_{n}}=\bar{A}^{(n)} \bar{\phi} \equiv A^{(n)}(\bar{u}, \lambda) \bar{\phi},
\end{gathered}
$$

and this $\bar{u}$ satisfies the $\mathrm{n}$-th KdV equation (2.9). It is found from (3.8), (2.8) and (3.10) that

$$
\begin{gathered}
\bar{\phi}_{t_{n}}=\bar{A}^{(n)} \bar{\phi}=\left[\phi-\frac{f}{\partial^{-1} f^{2}} \partial^{-1}(f \phi)\right]_{t_{n}} \\
=A^{(n)} \phi-\frac{1}{\partial^{-1} f^{2}}\left\{\left[A^{(n)} f-\frac{2 f}{\partial^{-1} f^{2}} \partial^{-1}\left(f A^{(n)} f\right)\right] \partial^{-1}(f \phi)+f \partial^{-1}\left[f A^{(n)} \phi+\phi A^{(n)} f\right]\right\} .
\end{gathered}
$$

So the covariance of (2.1) and (2.8) with respect to the action of binary DT (3.8) leads to the following lemma. 
Lemma 3.2 If $u$ is the solution of the $n$-th $K d V$ equation (2.9 and $f$ is a solution of (2.1) and (2.8) with $\lambda=\xi$ and the binary DT is given by (3.8), then the formula (3.11) holds.

(3) The second binary Darboux transformation.

Also the combination of $\tilde{g}$ and $\tilde{g}_{1}$ gives a solution of (3.2) and (3.3) with $\lambda=\xi$

$$
\tilde{g}_{2}=\tilde{g}+\tilde{g}_{1}=\frac{1}{f}\left(1+\partial^{-1} f^{2}\right) .
$$

By using $f$ and $\tilde{g}_{2}$, performing two-times repeated DT (3.1) leads to second binary Darboux transformation

$$
\begin{gathered}
\bar{\phi}=\frac{1}{\xi-\lambda}\left[\tilde{\phi}_{x}-\frac{\tilde{g}_{2 x}}{\tilde{g}_{2}} \tilde{\phi}\right] \\
=\frac{1}{\xi-\lambda}\left[\xi \phi-\lambda \phi+\frac{f}{1+\partial^{-1} f^{2}}\left(f_{x} \phi-f \phi_{x}\right)\right]=\phi-\frac{f}{1+\partial^{-1} f^{2}} \partial^{-1}(f \phi), \\
\bar{u}=\tilde{u}+2 \partial^{2} \ln \tilde{g}_{2}=u+2 \partial^{2} \ln \left(1+\partial^{-1} f^{2}\right) .
\end{gathered}
$$

Also the equation (2.1) and (2.8) are covariant with respect to the action of the binary DT (3.13), namely $\bar{\phi}, \bar{u}$ satisfy (3.9) and (3.10), $\bar{u}$ satisfies the $\mathrm{n}$-th KdV equation (2.9). Similarly, the covariance of (2.1) and (2.8) with respect to the action of binary DT (3.13) leads to the following lemma.

Lemma 3.3 If $u$ is a solution of the $n$-th $K d V$ equation 2.9 and $f$ is a solution of 2.1) and (2.8) with $\lambda=\xi$ and the binary DT is given by (3.13), then the following formula holds

$$
\begin{gathered}
\bar{\phi}_{t_{n}}=\bar{A}^{(n)} \bar{\phi}=\left[\phi-\frac{f}{1+\partial^{-1} f^{2}} \partial^{-1}(f \phi)\right]_{t_{n}} \\
=A^{(n)} \phi-\frac{1}{1+\partial^{-1} f^{2}}\left\{\left[A^{(n)} f-\frac{2 f}{1+\partial^{-1} f^{2}} \partial^{-1}\left(f A^{(n)} f\right)\right] \partial^{-1}(f \phi)+f \partial^{-1}\left[f A^{(n)} \phi+\phi A^{(n)} f\right]\right\} .
\end{gathered}
$$

\section{The Darboux transformation for the KdV hierarchy with self-consistent sources}

Based on the Darboux transformation for the KdV hierarchy, we now construct Darboux transformation and two binary Darboux transformations for the KdV hierarchy with self-consistent sources. The first binary Darboux transformation is an auto-Bäcklund transformation for the $\mathrm{n}$-th $\mathrm{KdV}$ equation with self-consistent sources (2.13). The second one is a Bäcklund transformation relating two n-th $\mathrm{KdV}$ equations with self-consistent sources (2.13) with degree $N$ and $N+1$, respectively.

(1) Darboux transformation for the KdV hierarchy with sources. 
Theorem 4.1 Assume that $u, \phi_{1}, \ldots, \phi_{N}$ be the solution of the $n$-th $K d V$ equation with selfconsistent sources (2.13) and $f_{1}$ satisfies (2.160) and (2.18) with $\lambda=\xi_{1}$, then the Darboux transformation is defined by

$$
\begin{gathered}
\bar{\psi}=\psi_{x}-\frac{f_{1, x}}{f_{1}} \psi, \\
\bar{u}=u+2 \partial^{2} \ln f_{1}, \\
\bar{\phi}_{j}=\frac{1}{\sqrt{\lambda_{j}-\xi_{1}}}\left[\phi_{j, x}-\frac{f_{1, x}}{f_{1}} \phi_{j}\right], \quad j=1, \ldots, N,
\end{gathered}
$$

the Lax representation (2.16a) and (2.18) are covariant with respect to the Darboux transformation (4.1). Namely $\bar{u}, \bar{\psi}, \bar{\phi}_{j}, j=1, \ldots, N$, satisfy

$$
\begin{gathered}
\bar{\psi}_{x x}+(\lambda+\bar{u}) \bar{\psi}=0 \\
\bar{\psi}_{t_{n}}=\bar{Q}^{(n, N)} \bar{\psi}=\bar{A}^{(n)} \bar{\psi}+\eta \bar{\psi}+\bar{B}_{N} \bar{\psi} \equiv A^{(n)}(\bar{u}, \lambda) \bar{\psi}+\eta \bar{\psi}+\alpha \sum_{j=1}^{N} \bar{\phi}_{j} \partial^{-1}\left(\bar{\phi}_{j} \bar{\psi}\right),
\end{gathered}
$$

and $\bar{u}, \bar{\phi}_{1}, \cdots, \bar{\phi}_{N}$ satisfy the $n$-th $K d V$ equation with self-consistent sources 2.13)

$$
\begin{gathered}
\bar{u}_{t_{n}}=D\left[-2 \bar{b}_{n+2}-2 \alpha \sum_{j=1}^{N} \bar{\phi}_{j}^{2}\right], \\
\bar{\phi}_{j, x x}+\left(\lambda_{j}+\bar{u}\right) \bar{\phi}_{j}=0, \quad j=1, \cdots, N .
\end{gathered}
$$

Proof Based on the results in the previous section, it is obvious that (4.2) and (4.4b) hold. In order to prove the (4.3) we need to show the following equality

$$
\tilde{\psi}_{t_{n}}=\left[\psi_{x}-\frac{f_{1, x}}{f_{1}} \psi\right]_{t_{n}}=\left(Q^{(n, N)} \psi\right)_{x}-\left(\frac{Q^{(n, N)} f_{1}}{f_{1}}\right)_{x} \psi-\frac{f_{1, x}}{f_{1}} Q^{(n, N)} \psi=\tilde{Q}^{(n, N)} \tilde{\psi}
$$

The Lemma 3.1 implies that equality (3.4) with $\phi$ replaced by $\psi$ holds. So we only need to check the terms containing $\phi_{1}, \ldots, \phi_{N}$ in the equality (4.5), i.e., to show the following equality

$$
\left(B_{N} \psi\right)_{x}-\left(\frac{B_{N} f_{1}}{f_{1}}\right)_{x} \psi-\frac{f_{1, x}}{f_{1}} B_{N} \psi=\bar{B}_{N} \bar{\psi}
$$

Using (4.1) and (2.17), we have

$$
\begin{gathered}
\bar{B}_{N} \bar{\psi}=\alpha \sum_{j=1}^{N} \bar{\phi}_{j} \partial^{-1}\left[\frac{1}{\sqrt{\lambda_{j}-\xi_{1}}}\left(\phi_{j, x}-\frac{f_{1, x}}{f_{1}} \phi_{j}\right)\left(\psi_{x}-\frac{f_{1, x}}{f_{1}} \psi\right)\right] \\
=\alpha \sum_{j=1}^{N} \frac{1}{\sqrt{\lambda_{j}-\xi_{1}}} \bar{\phi}_{j}\left[\phi_{j, x} \psi-\partial^{-1}\left(\phi_{j, x x} \psi\right)-\frac{f_{1, x}}{f_{1}} \phi_{j} \psi+\partial^{-1}\left(\frac{f_{1, x x}}{f_{1}} \phi_{j} \psi\right)\right] \\
=\alpha \sum_{j=1}^{N} \frac{1}{\sqrt{\lambda_{j}-\xi_{1}}} \bar{\phi}_{j}\left[\phi_{j, x} \psi-\frac{f_{1, x}}{f_{1}} \phi_{j} \psi+\left(\lambda_{j}-\xi_{1}\right) \partial^{-1}\left(\phi_{j} \psi\right)\right],
\end{gathered}
$$


and

the left terms in $(4.6)=\alpha \sum_{j=1}^{N}\left[\phi_{j, x} \partial^{-1}\left(\phi_{j} \psi\right)-\frac{1}{f_{1}} \psi \phi_{j, x} \partial^{-1}\left(f_{1} \phi_{j}\right)+\frac{f_{1, x}}{f_{1}^{2}} \psi \phi_{j} \partial^{-1}\left(f_{1} \phi_{j}\right)\right.$

$$
\begin{gathered}
\left.-\frac{f_{1, x}}{f_{1}} \phi_{j} \partial^{-1}\left(\phi_{j} \psi\right)\right]=\alpha \sum_{j=1}^{N} \sqrt{\lambda_{j}-\xi_{1}} \bar{\phi}_{j}\left[\partial^{-1}\left(\phi_{j} \psi\right)-\frac{1}{f_{1}} \psi \partial^{-1}\left(f_{1} \phi_{j}\right)\right] \\
=\alpha \sum_{j=1}^{N} \sqrt{\lambda_{j}-\xi_{1}} \bar{\phi}_{j}\left[\partial^{-1}\left(\phi_{j} \psi\right)+\frac{1}{f_{1}\left(\lambda_{j}-\xi_{1}\right)} \psi\left(f_{1} \phi_{j, x}-f_{1, x} \phi_{j}\right)\right] .
\end{gathered}
$$

Comparing (4.7) with (4.8), it is immediately found that equality (4.6) holds. The equation (4.2) and (4.3) lead to (4.4a). This completes the proof.

(2) The first binary Darboux transformation for the KdV hierarchy with sources.

Theorem 4.2 Assume that $u, \phi_{1}, \ldots, \phi_{N}$ be the solution of the $n$-th $K d V$ equation with selfconsistent sources (2.13) and $f_{1}$ satisfies (2.160) and (2.18) with $\lambda=\xi_{1}$, then the first binary Darboux transformation is defined by

$$
\begin{gathered}
\bar{\psi}=\psi-\frac{f_{1}}{\partial^{-1} f_{1}^{2}} \partial^{-1}\left(f_{1} \psi\right), \\
\bar{u}=u+2 \partial^{2} \ln \left(\partial^{-1} f_{1}^{2}\right), \\
\bar{\phi}_{j}=\phi_{j}-\frac{f_{1}}{\partial^{-1} f_{1}^{2}} \partial^{-1}\left(f_{1} \phi_{j}\right), \quad j=1, \ldots, N,
\end{gathered}
$$

the Lax representation (2.160) and (2.18) are covariant with respect to the binary Darboux transformation (4.9). Namely $\bar{u}, \bar{\psi}, \bar{\phi}_{j}, j=1, \ldots, N$, satisfy (4.9), (4.3) and the $n$-th $K d V$ equation with self-consistent sources (4.4).

Proof It is obvious that (4.2) and (4.4b) hold. Similarly, in order to prove the (4.3) we need to show the equality (3.11) with $\phi, A^{(n)}$ replaced by $\psi, Q^{(n, N)}$. In fact, using Lemma 3.2 , we only need to check the terms containing $\phi_{1}, \ldots, \phi_{N}$ in the equality, i.e., to show the following equality

$$
\begin{gathered}
B_{N} \psi-\frac{1}{\partial^{-1} f_{1}^{2}}\left[B_{N} f_{1}-2 \frac{1}{\partial^{-1} f_{1}^{2}} f_{1} \partial^{-1}\left(f_{1} B_{N} f_{1}\right)\right] \partial^{-1}\left(f_{1} \psi\right) \\
-\frac{f_{1}}{\partial^{-1} f_{1}^{2}} \partial^{-1}\left[f_{1} B_{N} \psi+\psi B_{N} f_{1}\right]=\bar{B}_{N} \bar{\psi}
\end{gathered}
$$

Notice that

$$
\begin{gathered}
\partial^{-1}\left[\frac{f_{1}^{2}}{\left(\partial^{-1} f_{1}^{2}\right)^{2}}\left(\partial^{-1}\left(f_{1} \phi_{j}\right)\right)\left(\partial^{-1}\left(f_{1} \psi\right)\right)\right]=-\frac{1}{\partial^{-1} f_{1}^{2}}\left(\partial^{-1}\left(f_{1} \phi_{j}\right)\right)\left(\partial^{-1}\left(f_{1} \psi\right)\right) \\
+\partial^{-1}\left[\frac{f_{1} \phi_{j}}{\partial^{-1} f_{1}^{2}} \partial^{-1}\left(f_{1} \psi\right)+\frac{f_{1} \psi}{\partial^{-1} f_{1}^{2}} \partial^{-1}\left(f_{1} \phi_{j}\right)\right] .
\end{gathered}
$$


Using (4.9) and (4.11), we have

$$
\begin{gathered}
\bar{B}_{N} \bar{\psi}=\alpha \sum_{j=1}^{N} \bar{\phi}_{j} \partial^{-1}\left(\bar{\phi}_{j} \bar{\psi}\right) \\
=\alpha \sum_{j=1}^{N} \bar{\phi}_{j} \partial^{-1}\left[\left(\phi_{j}-\frac{f_{1}}{\partial^{-1} f_{1}^{2}} \partial^{-1}\left(f_{1} \phi_{j}\right)\right)\left(\psi-\frac{f_{1}}{\partial^{-1} f_{1}^{2}} \partial^{-1}\left(f_{1} \psi\right)\right)\right] \\
=\alpha \sum_{j=1}^{N} \bar{\phi}_{j}\left[\partial^{-1}\left(\phi_{j} \psi\right)-\frac{1}{\partial^{-1} f_{1}^{2}}\left(\partial^{-1}\left(f_{1} \phi_{j}\right)\right)\left(\partial^{-1}\left(f_{1} \psi\right)\right)\right]
\end{gathered}
$$

and

$$
\begin{aligned}
& \text { the left terms in }(4.10)=\alpha \sum_{j=1}^{N}\left\{\phi_{j} \partial^{-1}\left(\phi_{j} \psi\right)-\frac{1}{\partial^{-1} f_{1}^{2}}\left[\phi_{j}\left(\partial^{-1}\left(f_{1} \phi_{j}\right)\right)\left(\partial^{-1}\left(f_{1} \psi\right)\right)\right.\right. \\
& \begin{array}{c}
\left.\left.-2 \frac{f_{1}}{\partial^{-1} f_{1}^{2}} \partial^{-1}\left(f_{1} \phi_{j} \partial^{-1}\left(f_{1} \phi_{j}\right)\right)\left(\partial^{-1}\left(f_{1} \psi\right)\right)+f_{1} \partial^{-1}\left(f_{1} \phi_{j} \partial^{-1}\left(\phi_{j} \psi\right)\right)+f_{1} \partial^{-1}\left(\phi_{j} \psi \partial^{-1}\left(\phi_{j} f_{1}\right)\right)\right]\right\} \\
=\alpha \sum_{j=1}^{N}\left[\phi_{j} \partial^{-1}\left(\phi_{j} \psi\right)-\frac{\phi_{j}}{\partial^{-1} f_{1}^{2}}\left(\partial^{-1}\left(f_{1} \phi_{j}\right)\right)\left(\partial^{-1}\left(f_{1} \psi\right)\right)\right. \\
\left.+\frac{f_{1}}{\left(\partial^{-1} f_{1}^{2}\right)^{2}}\left(\partial^{-1}\left(f_{1} \phi_{j}\right)\right)^{2}\left(\partial^{-1}\left(f_{1} \psi\right)\right)-\frac{f_{1}}{\partial^{-1} f_{1}^{2}}\left(\partial^{-1}\left(f_{1} \phi_{j}\right)\right)\left(\partial^{-1}\left(\phi_{j} \psi\right)\right)\right] .
\end{array}
\end{aligned}
$$

By substituting (4.9c) into (4.12) and comparing it with (4.13), it is immediately found that equality (4.10) holds. The equation (4.2) and (4.3) lead to (4.4a). This completes the proof.

(3) The second binary Darboux transformation for the KdV hierarchy with sources.

Theorem 4.3 Assume that $u, \phi_{1}, \ldots, \phi_{N}$ be the solution of the $n$-th $K d V$ equation with selfconsistent sources (2.13), $f_{1} \equiv \phi_{N+1}$ satisfies (2.160) and (2.18) with $\lambda=\lambda_{N+1}$ and $\eta=$ $-\frac{1}{2} \alpha$, then the second binary Darboux transformation is defined by

$$
\begin{gathered}
\bar{\psi}=\psi-\frac{f_{1}}{1+\partial^{-1} f_{1}^{2}} \partial^{-1}\left(f_{1} \psi\right)=\psi-\bar{\phi}_{N+1} \partial^{-1}\left(f_{1} \psi\right), \\
\bar{u}=u+2 \partial^{2} \ln \left(1+\partial^{-1} f_{1}^{2}\right), \\
\bar{\phi}_{j}=\phi_{j}-\frac{f_{1}}{1+\partial^{-1} f_{1}^{2}} \partial^{-1}\left(f_{1} \phi_{j}\right)=\phi_{j}-\bar{\phi}_{N+1} \partial^{-1}\left(f_{1} \phi_{j}\right), \quad j=1, \ldots, N,
\end{gathered}
$$

where

$$
\bar{\phi}_{N+1}=\frac{f_{1}}{1+\partial^{-1} f_{1}^{2}}, \quad f_{1}=\phi_{N+1},
$$

and the binary Darboux transformation (4.14) transforms the Lax representation (2.16a) and (2.18) with $\eta=-\frac{1}{2} \alpha$ into the following Lax representation

$$
\begin{gathered}
\bar{\psi}_{x x}+(\lambda+\bar{u}) \bar{\psi}=0 \\
\bar{\psi}_{t_{n}}=\bar{Q}^{(n, N+1)} \bar{\psi}=\bar{A}^{(n)} \bar{\psi}-\frac{1}{2} \alpha \bar{\psi}+\bar{B}_{N+1} \bar{\psi} \equiv A^{(n)}(\bar{u}, \lambda) \bar{\psi}-\frac{1}{2} \alpha \bar{\psi}+\alpha \sum_{j=1}^{N+1} \bar{\phi}_{j} \partial^{-1}\left(\bar{\phi}_{j} \bar{\psi}\right),
\end{gathered}
$$


and $\bar{u}, \bar{\phi}_{1}, \cdots, \bar{\phi}_{N+1}$ satisfies the $n$-th $K d V$ equation with self-consistent sources (2.13) with degree $N+1$

$$
\begin{gathered}
\bar{u}_{t_{n}}=D\left[-2 \bar{b}_{n+2}-2 \alpha \sum_{j=1}^{N+1} \bar{\phi}_{j}^{2}\right] \\
\bar{\phi}_{j, x x}+\left(\lambda_{j}+\bar{u}\right) \bar{\phi}_{j}=0, \quad j=1, \cdots, N+1 .
\end{gathered}
$$

Proof It is easy to see that (4.14d) holds for $j=N+1$. So, based on the results in previous section, it is obvious that $(4.15)$ and $(4.17 \mathrm{~b})$ hold. Similarly, in order to prove (4.16), by using Lemma 3.3 one only needs to check the terms containing $\phi_{1}, \ldots, \phi_{N}, \bar{\phi}_{N+1}$ in the equality (3.14) with $\phi, A^{(n)}$ replaced by $\psi, Q^{(n, N)}$, i.e., to show the following equality

$$
\begin{gathered}
B_{N} \psi-\frac{1}{1+\partial^{-1} f_{1}^{2}}\left[B_{N} f_{1}-\frac{1}{2} \alpha \bar{\phi}_{N+1}\left(1-\partial^{-1} f_{1}^{2}\right)-2 \bar{\phi}_{N+1} \partial^{-1}\left(f_{1} B_{N} f_{1}\right)\right] \partial^{-1}\left(f_{1} \psi\right) \\
-\bar{\phi}_{N+1} \partial^{-1}\left[\psi B_{N} f_{1}-\frac{1}{2} \alpha f_{1} \psi+f_{1} B_{N} \psi\right]=\bar{B}_{N+1} \bar{\psi}
\end{gathered}
$$

Notice that

$$
\partial^{-1}\left(\bar{\phi}_{N+1} \bar{\psi}\right)=\partial^{-1}\left[\frac{f_{1}}{1+\partial^{-1} f_{1}^{2}} \psi-\left(\frac{f_{1}}{1+\partial^{-1} f_{1}^{2}}\right)^{2} \partial^{-1}\left(f_{1} \psi\right)\right]=\frac{1}{1+\partial^{-1} f_{1}^{2}} \partial^{-1}\left(f_{1} \psi\right) .
$$

By means of (4.11) and (4.12) with $\partial^{-1} f_{1}^{2}$ replaced by $\left(1+\partial^{-1} f_{1}^{2}\right)$ one gets

$$
\begin{gathered}
\bar{B}_{N+1} \bar{\psi}=\alpha \sum_{j=1}^{N+1} \bar{\phi}_{j} \partial^{-1}\left(\bar{\phi}_{j} \bar{\psi}\right) \\
=\alpha \sum_{j=1}^{N} \bar{\phi}_{j}\left[\partial^{-1}\left(\phi_{j} \psi\right)-\frac{1}{1+\partial^{-1} f_{1}^{2}}\left(\partial^{-1}\left(f_{1} \phi_{j}\right)\right) \partial^{-1}\left(f_{1} \psi\right)\right]+\frac{\alpha \bar{\phi}_{N+1}}{1+\partial^{-1} f_{1}^{2}} \partial^{-1}\left(f_{1} \psi\right) .
\end{gathered}
$$

Using (4.13) with $\partial^{-1} f_{1}^{2}$ replaced by $\left(1+\partial^{-1} f_{1}^{2}\right)$ it is found

$$
\begin{aligned}
& \text { the left terms in }(4.18)=\alpha \sum_{j=1}^{N}\left\{\phi_{j} \partial^{-1}\left(\phi_{j} \psi\right)-\frac{1}{1+\partial^{-1} f_{1}^{2}}\left[\phi_{j}\left(\partial^{-1}\left(f_{1} \phi_{j}\right)\right) \partial^{-1}\left(f_{1} \psi\right)\right.\right. \\
& \left.\left.-\bar{\phi}_{N+1}\left(\partial^{-1}\left(f_{1} \phi_{j}\right)\right)^{2} \partial^{-1}\left(f_{1} \psi\right)\right]-\bar{\phi}_{N+1}\left(\partial^{-1}\left(f_{1} \phi_{j}\right)\right) \partial^{-1}\left(\phi_{j} \psi\right)\right\}+\frac{\alpha \bar{\phi}_{N+1}}{1+\partial^{-1} f_{1}^{2}} \partial^{-1}\left(f_{1} \psi\right)
\end{aligned}
$$

By substituting (4.140) into (4.20) and comparing it with (4.21), it is easy to see that equality (4.18) holds. The equations (4.15) and (4.16) yield (4.17). This completes the proof.

Remark The binary Darboux transformation defined by (4.14) is a non auto-Bäcklund transformation relating the two n-th KdV equations with self-consistent sources (2.13) and (4.17). This Darboux transformation can be used to construct the soliton solution for (2.13).

For example, in order to find one soliton solution for the $\mathrm{KdV}$ equation with self-consistent sources (2.19) with $N=1$, we start from the solution $u=0$ for the KdV equation with selfconsistent sources (2.19) with $N=0$. The solution for (2.20) with $N=0, u=0, \lambda=$ $-k^{2}, k>0, \eta=-\frac{1}{2} \alpha$ reads

$$
\phi_{1}=c e^{k x-k^{3} t-\frac{1}{2} \alpha t}
$$


Then one finds from (4.14) that

$$
\begin{aligned}
\bar{u} & =2 k^{2} \operatorname{sech}^{2}\left(k x-k^{3} t-\frac{1}{2} \alpha t+x_{0}\right), \\
\phi_{1} & =\frac{1}{2} \sqrt{2 k} \operatorname{sech}\left(k x-k^{3} t-\frac{1}{2} \alpha t+x_{0}\right),
\end{aligned}
$$

which is the one soliton solution for the KdV equation with self-consistent sources (2.19) with $N=1$.

\section{The m-times repeated binary Darboux transforma- tion for the KdV hierarchy with self-consistent sources}

It is evident that the Darboux transformation can be applied to (4.2), (4.3) and (4.15), (4.16) once more to produce some new solutions for the KdV hierarchy with self-consistent sources.

(1) The m-times repeated second binary Darboux transformation.

Assume that $f_{1}, \ldots, f_{m}$ be solution of (2.16a) and (2.18) with $\lambda=\lambda_{N+1}, \ldots, \lambda_{N+m}$, respectively. We use $u[i], \psi[i], f_{j}[i], \phi_{j}[i]$ to denote the action of i-times repeated binary Darboux transformation of (4.14) on the initial solution $u, \psi, f_{j}, \phi_{j}$. We have

$$
\begin{gathered}
f_{j}[i]_{x x}+\left(\lambda_{j}+u[i]\right) f_{j}[i]=0, \\
f_{j, t_{n}}[i]=Q^{(n, N+i)}[i] f_{j}[i] .
\end{gathered}
$$

We define two integral types of the Wronskian determinant of $k$ functions $g_{1}, \ldots, g_{k}$ in a similar way as in [27] by

$$
W_{1}\left(g_{1}, \ldots, g_{k}\right)=\operatorname{det} F, \quad W_{2}\left(g_{1}, \ldots, g_{k}\right)=\operatorname{det} G,
$$

where

$$
\begin{gathered}
F_{i j}=\delta_{i j}+\partial^{-1}\left(g_{i} g_{j}\right), \quad i, j=1, \ldots, k \\
G_{i j}=\delta_{i j}+\partial^{-1}\left(g_{i} g_{j}\right), \quad i=1, \ldots, k-1, \quad j=1, \ldots, k, \quad G_{k j}=g_{j}, \quad j=1, \ldots, k .
\end{gathered}
$$

Lemma 5.1 For arbitrary integers $l, k(1 \leq l \leq m-1,1 \leq k \leq m-l)$, we have

$$
\begin{gathered}
W_{1}\left(f_{l+1}[l], \ldots, f_{l+k}[l]\right)=\frac{W_{1}\left(f_{l}[l-1], f_{l+1}[l-1], \ldots, f_{l+k}[l-1]\right)}{1+\partial^{-1} f_{l}^{2}[l-1]}, \\
W_{2}\left(f_{l+1}[l], \ldots, f_{l+k}[l], \psi[l]\right)=\frac{W_{1}\left(f_{l}[l-1], f_{l+1}[l-1], \ldots, f_{l+k}[l-1], \psi[l-1]\right)}{1+\partial^{-1} f_{l}^{2}[l-1]} .
\end{gathered}
$$


Proof According to (4.14), we have

$$
f_{l+j}[l]=f_{l+j}[l-1]-\frac{f_{l}[l-1]}{1+\partial^{-1} f_{l}^{2}[l-1]} \partial^{-1}\left(f_{l}[l-1] f_{l+j}[l-1]\right),
$$

so using (4.11)

$$
\begin{gathered}
F_{i j}=\delta_{i j}+\partial^{-1}\left(f_{l+i}[l] f_{l+j}[l]\right)=\delta_{i j}+\partial^{-1}\left(f_{l+i}[l-1] f_{l+j}[l-1]\right) \\
-\frac{1}{1+\partial^{-1} f_{l}^{2}[l-1]}\left(\partial^{-1} f_{l+i}[l-1] f_{l}[l-1]\right)\left(\partial^{-1} f_{l}[l-1] f_{l+j}[l-1]\right) \equiv \delta_{i j}+a_{i j}-b a_{i 0} a_{0 j},
\end{gathered}
$$

where

$$
a_{i j}=\partial^{-1}\left(f_{l+i}[l-1] f_{l+j}[l-1]\right), \quad b=\frac{1}{1+\partial^{-1} f_{l}^{2}[l-1]} .
$$

Then

$$
\begin{aligned}
& W_{1}\left(f_{l+1}[l], \ldots, f_{l+k}[l]\right)=\operatorname{det}\left(F_{i j}\right) \\
& =\left(\begin{array}{ccccc}
1+a_{11}-b a_{10} a_{01} & a_{12}-b a_{10} a_{02} & a_{13}-b a_{10} a_{03} & \cdots & a_{1 k}-b a_{10} a_{0 k} \\
a_{21}-b a_{20} a_{01} & 1+a_{22}-b a_{20} a_{02} & a_{23}-b a_{20} a_{03} & \cdots & a_{2 k}-b a_{20} a_{0 k} \\
\vdots & \vdots & \vdots & \ddots & \vdots \\
a_{k 1}-b a_{k 0} a_{01} & a_{k 2}-b a_{k 0} a_{02} & a_{k 3}-b a_{k 0} a_{03} & \cdots & 1+a_{k k}-b a_{k 0} a_{0 k}
\end{array}\right) \\
& =\left(\begin{array}{cccc}
1+a_{11} & a_{12} & \cdots & a_{1 k} \\
a_{21} & 1+a_{22} & \cdots & a_{2 k} \\
\vdots & \vdots & \ddots & \vdots \\
a_{k 1} & a_{k 2} & \cdots & 1+a_{k k}
\end{array}\right)-b a_{01}\left(\begin{array}{ccccc}
a_{10} & a_{12} & a_{13} & \cdots & a_{1 k} \\
a_{20} & 1+a_{22} & a_{23} & \cdots & a_{2 k} \\
\vdots & \vdots & \vdots & \ddots & \vdots \\
a_{k 0} & a_{k 2} & a_{k 3} & \cdots & 1+a_{k k}
\end{array}\right) \\
& -b a_{02}\left(\begin{array}{ccccc}
1+a_{11} & a_{10} & a_{13} & \cdots & a_{1 k} \\
a_{21} & a_{20} & a_{23} & \cdots & a_{2 k} \\
\vdots & \vdots & \vdots & \ddots & \vdots \\
a_{k 1} & a_{k 0} & a_{k 3} & \cdots & 1+a_{k k}
\end{array}\right)-\cdots \\
& -b a_{0 k}\left(\begin{array}{ccccc}
1+a_{11} & a_{12} & \cdots & a_{1(k-1)} & a_{10} \\
a_{21} & 1+a_{22} & \cdots & a_{2(k-1)} & a_{20} \\
\vdots & \vdots & \ddots & \vdots & \vdots \\
a_{k 1} & a_{k 2} & \cdots & a_{k(k-1)} & a_{k 0}
\end{array}\right) \\
& =\frac{1}{1+a_{00}}\left(\begin{array}{ccccc}
1+a_{00} & a_{01} & a_{02} & \cdots & a_{0 k} \\
a_{10} & 1+a_{11} & a_{12} & \cdots & a_{1 k} \\
\vdots & \vdots & \vdots & \ddots & \vdots \\
a_{k 0} & a_{k 1} & a_{k 2} & \cdots & 1+a_{k k}
\end{array}\right)=\frac{W_{1}\left(f_{l}[l-1], \ldots, f_{l+k}[l-1]\right)}{1+\partial^{-1} f_{l}^{2}[l-1]} .
\end{aligned}
$$

In the similar way the formula (5.4) can be proved. This completes the proof. 
Theorem 5.1 Assume that $u, \phi_{1}, \cdots \phi_{N}$ is solution of the $n$-th $K d V$ equation with selfconsistent sources (2.13), $f_{1}, \ldots, f_{m}$ be solution of (2.160) and (2.18) with $\lambda=\lambda_{N+1}, \ldots, \lambda_{N+m}$, respectively, and $\eta=-\frac{1}{2} \alpha$. Then the $m$-times repeated binary Darboux transformation of (4.14) is given by

$$
\begin{gathered}
\psi[m]=\frac{W_{2}\left(f_{1}, \ldots, f_{m}, \psi\right)}{W_{1}\left(f_{1}, \ldots, f_{m}\right)}, \\
u[m]=u+2 \partial^{2} \ln W_{1}\left(f_{1}, \ldots, f_{m}\right), \\
\phi_{j}[m]=\frac{W_{2}\left(f_{1}, \ldots, f_{m}, \phi_{j}\right)}{W_{1}\left(f_{1}, \ldots, f_{m}\right)}, \quad j=1, \ldots, N, \\
\phi_{N+j}[m]=\frac{W_{2}\left(f_{1}, \ldots, f_{m}, f_{j}\right)}{W_{1}\left(f_{1}, \ldots, f_{m}\right)}=\frac{W_{2}\left(f_{1}, \ldots, f_{j-1}, f_{j+1}, \ldots, f_{m}, f_{j}\right)}{W_{1}\left(f_{1}, \ldots, f_{m}\right)}, \quad j=1, \cdots, m,
\end{gathered}
$$

and $u[m], \psi[m], \phi_{1}[m], \cdots, \phi_{N+m}[m]$ satisfy

$$
\psi_{x x}[m]+(\lambda+u[m]) \psi[m]=0,
$$

$$
\psi_{t_{n}}[m]=Q^{(n, N+m)}[m] \psi[m]=A^{(n)}(u[m], \lambda) \psi[m]-\frac{1}{2} \alpha \psi[m]+\alpha \sum_{j=1}^{N+m} \phi_{j}[m] \partial^{-1}\left(\phi_{j}[m] \psi[m]\right),
$$

and

$$
\begin{gathered}
u_{t_{n}}[m]=D\left[-2 b_{n+2}(u[m])-2 \alpha \sum_{j=1}^{N+m} \phi_{j}^{2}[m]\right], \\
\phi_{j, x x}[m]+\left(\lambda_{j}+u[m]\right) \phi_{j}[m]=0, \quad j=1, \cdots, N+m .
\end{gathered}
$$

proof Using (4.14), (5.3) and (5.4), one obtains

$$
\begin{gathered}
\psi[m]=\psi[m-1]-\frac{f_{m}[m-1]}{1+\partial^{-1} f_{m}^{2}[m-1]} \partial^{-1}\left(f_{m}[m-1] \psi[m-1]\right) \\
=\frac{1}{1+\partial^{-1} f_{m}^{2}[m-1]} W_{2}\left(f_{m}[m-1], \psi[m-1]\right)=\frac{W_{2}\left(f_{m}[m-1], \psi[m-1]\right)}{W_{1}\left(f_{m}[m-1]\right)} \\
=\frac{W_{2}\left(f_{m-1}[m-2], f_{m}[m-2], \psi[m-2]\right)}{1+\partial^{-1} f_{m-1}^{2}[m-2]} \cdot \frac{1+\partial^{-1} f_{m-1}^{2}[m-2]}{W_{1}\left(f_{m-1}[m-2], f_{m}[m-2]\right)}=\cdots=\frac{W_{2}\left(f_{1}, \ldots, f_{m}, \psi\right)}{W_{1}\left(f_{1}, \ldots, f_{m}\right)}, \\
u[m]=u[m-1]+2 \partial^{2} \ln \left(1+\partial^{-1} f_{m}^{2}[m-1]\right)=u[m-1]+2 \partial^{2} \ln W_{1}\left(f_{m}[m-1]\right) \\
=u[m-2]+2 \partial^{2} \ln \left(1+\partial^{-1} f_{m-1}^{2}[m-2]\right)+2 \partial^{2} \ln \frac{W_{1}\left(f_{m-1}[m-2], f_{m}[m-2]\right)}{1+\partial^{-1} f_{m-1}^{2}[m-2]} \\
=u[m-2]+2 \partial^{2} \ln W_{1}\left(f_{m-1}[m-2], f_{m}[m-2]\right)=\cdots=u+2 \partial^{2} \ln W_{1}\left(f_{1}, \ldots, f_{m}\right) .
\end{gathered}
$$


Similarly we can prove the (5.7d) and (5.7d). It is easy to find (5.8), (5.9) and (5.10) from the Proposition 4.3.

The m-times repeated binary Darboux transformation (5.8) provides a Bäcklnud transformation relating two n-th $\mathrm{KdV}$ equations with self-consistent sources (2.13) with degree $N$ and $N+m$, respectively. We now use the N-times repeated binary Darboux transformation (5.7) to construct the N-soliton solution for the n-th KdV equation with self-consistent sources (2.13) with $\lambda_{j}=-k_{j}^{2}<0, k_{j}>0, j=1, \cdots, N$. We start from (2.13) with $N=0$. Taking $N=0, u=0, \lambda=-k_{j}^{2}, \eta=-\frac{1}{2} \alpha$, then (2.16a) and (2.18) reduce to

$$
\begin{gathered}
\psi_{x x}-k_{j}^{2} \psi=0, \\
\psi_{t_{n}}=(-1)^{n} k_{j}^{2 n} \psi_{x}-\frac{1}{2} \alpha \psi,
\end{gathered}
$$

which solution is given by

$$
f_{j}=e^{k_{j} x+(-1)^{n} k_{j}^{2 n+1} t_{n}-\frac{1}{2} \alpha t_{n}+x_{0, j}}, \quad j=1, \cdots, N .
$$

Then according to the Proposition 5.1, the N-soliton solution for the n-th KdV equation with self-consistent sources (2.13) with $\lambda_{j}=-k_{j}^{2}<0, k_{j}>0, j=1, \cdots, N$, is given by

$$
\begin{gathered}
u=2 \partial^{2} \ln W_{1}\left(f_{1}, \ldots, f_{N}\right), \\
\phi_{j}=\frac{W_{2}\left(f_{1}, \ldots, f_{N}, f_{j}\right)}{W_{1}\left(f_{1}, \ldots, f_{N}\right)}=\frac{W_{2}\left(f_{1}, \ldots, f_{j-1}, f_{j+1}, \ldots, f_{N}, f_{j}\right)}{W_{1}\left(f_{1}, \ldots, f_{N}\right)}, \quad j=1, \cdots, N,
\end{gathered}
$$

where $f_{j}$ is given by (5.13).

(2) The m-times repeated first binary Darboux transformation.

We define

$$
W_{1}\left(g_{1}, \ldots, g_{k}\right)=\operatorname{det} F, \quad W_{2}\left(g_{1}, \ldots, g_{k}\right)=\operatorname{det} G
$$

where

$$
\begin{gathered}
F_{i j}=\partial^{-1}\left(g_{i} g_{j}\right), \quad i, j=1, \ldots, k \\
G_{i j}=\partial^{-1}\left(g_{i} g_{j}\right), \quad i=1, \ldots, k-1, \quad j=1, \ldots, k, \quad G_{k j}=g_{j}, \quad j=1, \ldots, k .
\end{gathered}
$$

In the exactly same way we can prove the following theorem.

Theorem 5.2 Assume that $u, \phi_{1}, \cdots \phi_{N}$ is solution of the $n$-th $K d V$ equation with selfconsistent sources (2.13), $f_{1}, \ldots, f_{m}$ be the solutions of (2.160) and (2.18) with $\lambda=\xi_{1}, \ldots, \xi_{m}$, respectively. Then the m-times repeated binary Darboux transformation of (4.9) is given by

$$
\psi[m]=\frac{W_{2}\left(f_{1}, \ldots, f_{m}, \psi\right)}{W_{1}\left(f_{1}, \ldots, f_{m}\right)}
$$




$$
\begin{gathered}
u[m]=u+2 \partial^{2} \ln W_{1}\left(f_{1}, \ldots, f_{m}\right), \\
\phi_{j}[m]=\frac{W_{2}\left(f_{1}, \ldots, f_{m}, \phi_{j}\right)}{W_{1}\left(f_{1}, \ldots, f_{m}\right)}, \quad j=1, \cdots, N,
\end{gathered}
$$

and $u[m], \psi[m], \phi_{1}[m], \cdots, \phi_{N}[m]$ satisfy

$$
\begin{gathered}
\psi_{x x}[m]+(\lambda+u[m]) \psi[m]=0, \\
\psi_{t_{n}}[m]=Q^{(n, N)}[m] \psi[m],
\end{gathered}
$$

and

$$
\begin{gathered}
u_{t_{n}}[m]=D\left[-2 b_{n+2}(u[m])-2 \alpha \sum_{j=1}^{N} \phi_{j}^{2}[m]\right], \\
\phi_{j, x x}[m]+\left(\lambda_{j}+u[m]\right) \phi_{j}[m]=0, \quad j=1, \cdots, N .
\end{gathered}
$$

(3) The m-times repeated Darboux transformation of (4.1).

We define the Wronskian determinant $W$ by

$$
W_{1}\left(g_{1}, \ldots, g_{k}\right)=\operatorname{det} F, \quad F_{i j}=\frac{\partial^{i-1} g_{j}}{\partial x^{i-1}}, \quad i, j=1, \ldots, k .
$$

In the exactly same way we can prove the following theorem.

Theorem 5.3 Assume that $u, \phi_{1}, \cdots \phi_{N}$ is solution of the $n$-th $K d V$ equation with selfconsistent sources (2.19), $f_{1}, \ldots, f_{m}$ be the solutions of (2.160) and (2.18) with $\lambda=\xi_{1}, \ldots, \xi_{m}$, respectively. Then the m-times repeated Darboux transformation of (4.1) is given by

$$
\begin{gathered}
\psi[m]=\frac{W\left(f_{1}, \ldots, f_{m}, \psi\right)}{W\left(f_{1}, \ldots, f_{m}\right)}, \\
u[m]=u+2 \partial^{2} \ln W\left(f_{1}, \ldots, f_{m}\right), \\
\phi_{j}[m]=\frac{W\left(f_{1}, \ldots, f_{m}, \phi_{j}\right)}{W\left(f_{1}, \ldots, f_{m}\right)}, \quad j=1, \cdots, N,
\end{gathered}
$$

and $u[m], \psi[m], \phi_{1}[m], \cdots, \phi_{N}[m]$ satisfy (5.18), (5.19) and (5.20). 


\section{Acknowledgment}

This work was in part supported by a grant from the Research Grants Council of Hong Kong Special Administrative Region, China (Project no. 9040466), and a grant from the City

University of Hong Kong (Project no. 7001041), as well as by the Special Funds for Chinese Major Basic Research Project "Nonlinear Science" .

\section{References}

[1] V. K. Mel'nikov, Commun. Math. Phys. 120, 451 (1989);

[2] V. K. Mel'nikov, Commun. Math. Phys. 126, 201-215 (1989).

[3] V. K. Mel'nikov, Inverse Problem 6, 233-246 (1990).

[4] D. J. Kaup, Phys. Rev. Lett. 59, 2063 (1987).

[5] J. Leon and A. Latifi, J. Phys. A 23, 1385 (1990).

[6] C. Claude, A. Latifi and J. Leon, J. Math. Phys. 32, 3321 (1991).

[7] R. A. Vlasov and E. V. Doktorov E V, Dokl. Akad. Nauk BSSR 26, 17 (1991).

[8] E. V. Doktorov and R. A. Vlasov, Opt. Acta 30, 223 (1993).

[9] M. Nakazawa, E. Yomada and H. Kubota, Phys. Rev. Lett. 66, 2625 (1991).

[10] E. V. Doktorov and V. S. Shchesnovich, Phys. Lett. A 207, 153 (1995).

[11] V. S. Shchesnovich and E. V. Doktorov, Phys. Lett. A 213, 23 (1996).

[12] V. K. Mel'nikov, J. Math. Phys. 31, 1106 (1990).

[13] J. Leon, J. Math. Phys. 29, 2012 (1988); Phys. Lett. A 144, 444 (1990).

[14] M. Antonowicz and S. R. Wojciechowski, Phys. Lett. A 165, 47 (1992).

[15] Y. B. Zeng, J. Phys. A: Math. Gen. 26, L273 (1993).

[16] Y. B. Zeng, Physica D 73, 171 (1994).

[17] Y. B. Zeng and Y. S. Li, Acta Mathematica Sinica, New Series, 12, 217 (1996). 
[18] W. X. Ma and W. Strampp, Phys. Lett. A 185, 277 (1994).

[19] W. X. Ma, B. Fuchssteiner and W. Oevel, Physica A 233, 331 (1996).

[20] Y. B. Zeng, Physica A 262,405 (1999).

[21] V. K. Mel'nikov, Phys. Lett. A, 133, 493 (1988).

[22] V. K. Mel'nikov, Inverse Probl. 8, 133 (1992).

[23] Y. B. Zeng, W. X. Ma and R. L. Lin, J. Math. Phys. 41(8), 5453 (2000).

[24] V. B. Matveev and M. A. Salle, Darboux transformations and solitons (Berlin: Springer, 1991).

[25] V. E. Zakharov and A. B. Shabat, Funk. Anal. Appl. 8, 226 (1974).

[26] A. Yu Orlov and S. R. Wojciechowski, Physica D, 69, 77 (1993).

[27] Y. Chen and Mei A, Chinese Anal. A, 20, 667 (1999).

[28] A. C. Newell, Solitons in Mathematics and Physics(Philadelphia: SIAM, 1985)

[29] Y. B. Zeng, Phys. Lett. A 128, 488 (1991). 